\title{
Stark widths for astrophysically important ns-np transitions in Sc II, Y II and Zr II spectra ${ }^{\star}$
}

\author{
L.Č. Popović and M.S. Dimitrijević \\ Astronomical Observatory, Volgina 7, 11000 Belgrade, Yugoslavia \\ Received April 16; accepted April 22, 1996
}

\begin{abstract}
Here we present Stark widths for some astrophysically important ns-np transitions of Sc II, Y II and $\mathrm{Zr}$ II. Stark widths were calculated within the modified semiempirical approach. The obtained results were compared with simple estimates based on regularities and systematic trends.
\end{abstract}

Key words: atomic data — lines: profiles

\section{Introduction}

Spectral lines of Sc II, Y II and Zr II are present in spectra of hot stars, as e.g. in spectra of $\phi$ Her $\left(T_{\text {eff }} \approx\right.$ $11500 \mathrm{~K}, \log g=3.5)$ and $o$ And $\left(T_{\text {eff }} \approx 9500 \mathrm{~K}, \log g=3.5\right)$ (Adelman \& Lanz 1987) as well as in other hot stars (see e.g. Adelman et al. 1979; Redfors \& Cowley 1993; Kupka et al. 1994). Lines of these ions are also observed in the Solar spectrum (see e.g. Moore 1950, 1952; Krueger et al. 1968).

The electron impact broadening mechanism is the main pressure broadening mechanism in the hot star atmospheres $\left(T_{\text {eff }} \gtrsim 10000 \mathrm{~K}\right)$, and it is of interest especially for A type stars and white dwarfs. Moreover, Stark broadening of lines orginating from energy levels with high principal quantum numbers may be important and for some lines in atmospheres of cooler stars (see e.g. Vince et al. 1985).

In this paper we present Stark widths for 18 transitions of Sc II, Y II and Zr II, which are needed for stellar atmospheres investigations (Lanz \& Artru 1987).

\section{Results and discussions}

For the Stark broadening parameters calculation the modified semiempirical approach (Dimitrijević \& Konjević 1980 , for the case of complex heavy ions see also Popović \& Dimitrijević 1996) was used. Calculations were carried out for ns -np transitions from 6 multiplets for each of considered ions. We have selected only such multiplets where

Send offprint requests to: L.Č. Popović

*Tables only in electronic form can be accessed by ftp at cdsarc.u-strasbg.fr or 130.79.128.5 or on www at http://cdsweb.u-strasbg.fr/abstract.html it was possible to calculate Stark widths within the accuracy of the modified semiempirical approch. For these ions it is not possible to use more elaborate methods (as e.g. semiclassical one), because of the lack of atomic data.

The atomic data (energy levels) needed for calculations were taken from Moore (1971), Bashkin \& Stoner (1978) and Andersen et al. (1978). Oscillator strengths have been calculated by using Bates \& Damgaard (1949) method and the tables of Oertel \& Shomo (1968). For some lines of considered ions, the oscillator strengths from the Sollar and the stellar spectra (as e.g. Boyarchuk \& Boyarchuk 1960; Allen 1976), as well as determined theoretically and experimentally (see e.g. Andersen et al. 1978 and Malloy \& Czyzak 1978) have been used.

In Tables 1-3 Stark full widths for Sc II, Y II and Zr II, respectively, are presented. The data are given for an electron density of $10^{23} \mathrm{~m}^{-3}$ and temperatures from 5000 to $50000 \mathrm{~K}$.

In the case of these ions there is not measured or calculated Stark broadening parameters, only the simple estimates of Lakićević (1983) based on regularities and systematic trends for Sc II $\left(\mathrm{a}^{3} \mathrm{D}-\mathrm{z}^{3} \mathrm{~F}^{0}\right)$ and $\mathrm{Zr}$ II $\left(a^{4} \mathrm{~F}-\mathrm{z}^{4} \mathrm{G}^{0}\right)$ were published. Comparison of our calculated Stark widths with the estimates given by Lakićević (1983) are shown in Table 4.

As one can see, our calculated Stark widths are in good agremeent with the simple estimates of Lakićević (1983).

In order to show how much is important to take into account the Stark broadening effect for the modelling and analyses of hot star atmospheres, we have compared termal Doppler and Stark widths for Kurucz's (1979) model of an A type star with $\log g=4.0$ and $T_{\text {eff }}=10000$ K. In Figs. 1 and 2 termal Doppler and Stark widths are 


\begin{tabular}{|c|c|c|c|}
\hline Transition & $(\mathrm{FWHM})_{1}$ & $(\mathrm{FWHM})_{2}$ & $(\text { FWHM })_{1} /(\mathrm{FWHM})_{2}$ \\
\cline { 2 - 3 } & this paper & Lakićević $(1983)$ & \\
\hline Sc II $\left(\mathrm{a}^{3} \mathrm{D}-\mathrm{z}^{3} \mathrm{~F}^{0}\right)$ & $0.0107 \mathrm{~nm}$ & $0.0091 \mathrm{~nm}$ & 1.17 \\
Zr II $\left(\mathrm{a}^{4} \mathrm{~F}-\mathrm{z}^{4} \mathrm{G}^{0}\right)$ & $0.00761 \mathrm{~nm}$ & $0.0084 \mathrm{~nm}$ & 0.91 \\
\hline
\end{tabular}

presented as functions of temperature and optical depth, respectively, for an Y II line $(\lambda=437.9 \mathrm{~nm})$. One can see that exist conditions where Stark width is comparable and even up to one order of magnitude larger than the termal Doppler width. Consequently, one can see that exist conditions where Stark broadening may be principal broadening mechanism influencing line shape formation.

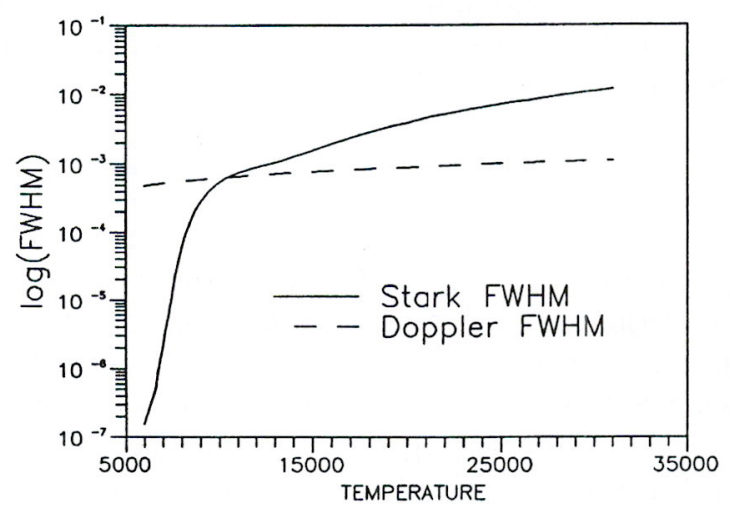

Fig. 1. Comparison of termal Doppler and Stark widths for $437.9 \mathrm{~nm}$ (Y II) line as a function of temperature for an A star $\left(T_{\text {eff }}=10000 \mathrm{~K}, \log g=4.0\right)$. The abundance of ytrium is as in Solar atmosphere

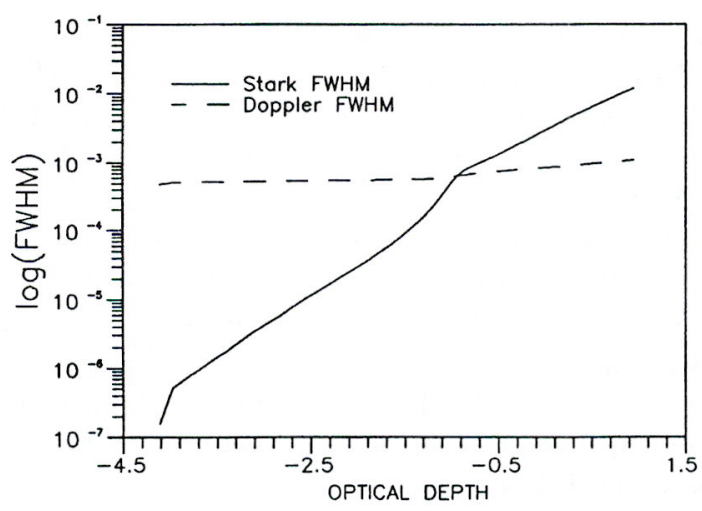

Fig. 2. Same as in Fig. 1, but the widths are presented as a function of optical depth
We hope that the presented results will be useful for astrophysical purposes.

\section{References}

Adelman S.J., Bidelman W.P., Pyper D.M., 1979, ApJS 40, 371

Adelman S.J., Lanz T., 1987, Elemental abundance analyses, the Institut d'Astronomie de l'Université de Lausanne

Allen M.S., 1976, PASP 88, 338

Andersen T., Ramanujam P.S., Bahr K., 1978, ApJ 223, 344

Bashkin S., Stoner J.O., 1987, Atomic energy levels \& Grotrian diagrams 2. North-Holland Publishing Company, Amsterdam - Oxford - New York

Bates D.R., Damgaard A., 1949, Phyl. Roy. Soc. London A 242, 101

Boyarchuk M.E., Boyarchuk A.A., 1960, Izv. Krymskoy Astrophys. Obs. 22, 234

Dimitrijević M.S., Konjević N., 1980, JQSRT 24, 451

Krueger T.K., Aller L.H., Ross J., Czyzak S.J., 1968, ApJ 152, 765

Kupka F., Ryabchikova T., Bolgova G., et al., 1994, In: Chemically peculiar and magnetic stars on and close to upper main sequence, Tatranska Lomnica, pp. 130-136

Kurucz R.L., 1979, ApJS 40, 1

Lakićević I.S., 1983, A\&A 127, 37

Lanz T., Artru M.-C., 1987, Elemental abundance analyses. In: Adelman S.J. and Lanz T. (eds.), the Institut d'Astronomie de l'Université de Lausanne, p. 156

Malloy P.J., Czyzak S.J., 1978, Astrophys. Space Sci. 58, 365

Moore C.E., 1950, An Ultraviolet Multplet Table, Circular of the NBS 488, Sect. 1, Washington D.C.

Moore C.E., 1952, An Ultraviolet Multplet Table, Circular of the NBS 488, Sect. 2, Washington D.C.

Moore C.E., 1971, Atomic energy levels, Nat. Stand. Ref. Data Ser., NBS, Vols. I, II, Washington D.C.

Oertel G.K., Shomo L.P., 1968, ApJS 16, 175

Popović L. C̆., Dimitrijević M.S., 1996, Phys. Scr. 53, 325

Redfors A., Cowley C.R., 1993, A\&A 271, 273

Vince I., Dimitrijević M.S., Kršljanin V., 1985, in Progres in Stellar Spectral Line Formation Theory. In: Beckman J., Crivellari L., Reidel D. (eds.). Dordrecht, p. 373 